\title{
Subtotal petrosectomy after recurrent otogenic meningitis. A case report and literature review
}

\author{
Enrico Maria Amadei* and Claudio Cola \\ Department of Otorhinolaryngology and Audiology, Infermi Hospital, Rimini, Italy
}

\begin{abstract}
Otogenic meningitis is a serious and fortunately rare complication of a middle ear otitis. Recurrent otogenic meningitis is even more rare and dangerous. This requires primarily the search for the cause. Therefore, it is indicated to perform an effective, safe and stable surgical technique. The purpose is to bring the patient to complete healing. This surgical technique is represented by subtotal petrosectomy.
\end{abstract}

\section{Introduction}

Subtotal petrosectomy (SP) in a well definited procedure consisting in a canal wall down mastoidectomy with removal of the ossicular chain, the obliteration of the Eustachian tube, the filling of the eardrum and mastoid with abdominal fat and the blind sac closure of the external auditory canal. This technique allows to isolate the middle ear from the external environment. Thus the passage of cerebrospinal fluid (CSF) from the central nervous system to the outside and vice versa of germs from the outside to the meninges are avoided.

\section{Case report}

We describe the case of a 37-year-old man with a recent abscess involving the brainstem and the right cerebellar peduncle. In the anamnesis, the patient reported a post-traumatic fracture of the right temporal bone at the age of 5 , resulting in a comatose state lasting 10 days. At the age of 6 he had a first episode of meningitis.

At the age of 37 the Patient $(\mathrm{Pt})$ presented a sudden headache and then a stiffness of the nuchal region after a trivial neglected right otitis media. A post-traumatic pathological pathway between middle ear and the posterior cranial fossa was evidenced with a urgent CT (Figure 1). The new otogenic meningitis was confirmed with a MRI (Figure 2), wich showed an abscess of the brainstem. This infection has regressed thanks to medical therapy alone.

We executed an audiometric exam, finding a right anacusia and a normal left hearing. Finally we performed a right SP (Figure 3). The Pt went to a speedy recovery, without complications. He did not present recurrences of otomastoiditis or meningitis or cerebral abscess after a 12-months follow-up (Figure 4).

\section{Discussion}

This Pt risked his life at least 2 times because of the fracture of the right petrous bone that occurred at a young age. We obtain a definitive cure of the problem performing an SP.

The classical indications for SP are recurrent chronic otitis with no concrete and realistic possibility of improving the auditory function (plurioperated patients without success); supralabyrinthine and
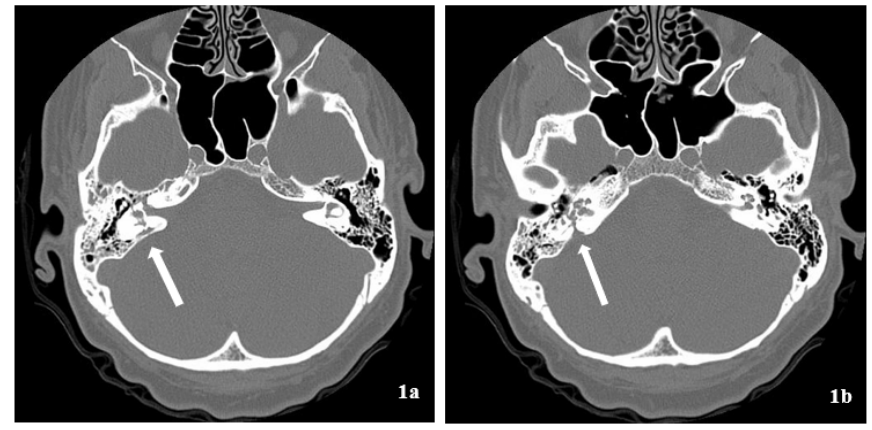

Figure 1. Pre-surgery CT without contrast: post-traumatic pathological pathway between middle ear (in front of the oval window), inner ear and posterior right cranial fossa. Look at arrows to see the transverse petrous temporal bone fracture

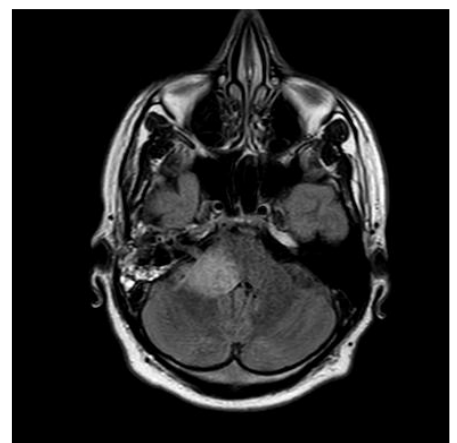

Figure 2. Pre-surgery MRI: right otitis media associated with an abscess involving the brainstem and the right cerebellar peduncle (the fistular connection between the middle ear and the ponto-cerebellar angle is clearly evident)

*Correspondence to: Enrico Maria Amadei, Department of Otorhinolaryngology and Audiology, Infermi Hospital, Rimini, via Popilia 233, Rimini, RN, 47922, Italy, E-mail: enricomaria.amadei@libero.it

Key words: subtotal petrosectomy, recurrent otogenic meningitis, temporal bone fracture

Received: March 17, 2019; Accepted: March 25, 2019; Published: March 28, 2019 


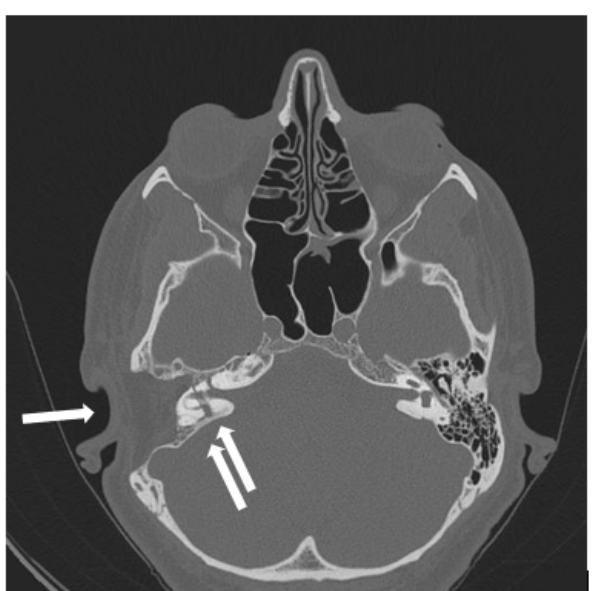

Figure 3. Post-surgery CT: Subtotal petrosectomy. We can see a canal wall down mastoidectomy with removal of the ossicular chain, and the filling of the eardrum and mastoid with abdominal fat (single arrow). Obviously we continue to see the pathological pathway of the right temporal bone (double arrows)

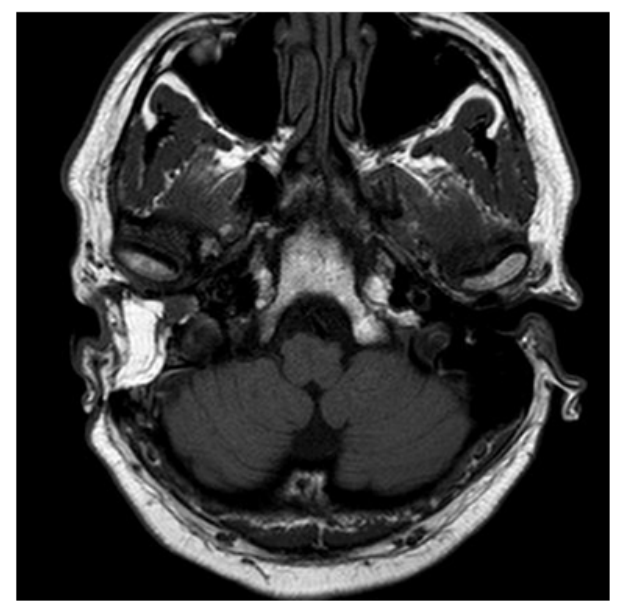

Figure 4. Post-surgery MRI: non-recurrence of right otomastoiditis or meningitis or cerebral abscess after a 12-months follow-upa

infralabyrinthine cholesteatoma; fractures of the temporal bone, as in this case; placement of a cochlear or middle ear implantations where an open cavity has previously been performed or in cases of congenital cochlear malformation where there is a high risk of a CSF gusher; in combination with another neuro-otologic procedure (transotic and infratemporal fossa approaches types $\mathrm{A}, \mathrm{B}$ or $\mathrm{C}$ ); finally in cases of osteoradionecrosis of the temporal bone.

Complications are rare. From the Literature we know that Pts can often meet a deterioration of the bone conduction hearing [1,2]. In this case the Pt had a right anacusia, therefore we have avoided the problem of a iatrogenic hearing impairment.

Other problem can be a postauricular wound fistula. In Literature this goes from $1.1 \%$ [2] to $6 \%$ [3] to $16 \%$ [4]. Lyutenski, et al. compared different reconstruction techniques: with or without temporalis muscle flap or with reinforcing material like polydioxanone foil or bovine pericardium or allogenic fascia lata for wound closure. They did not find significant differences in the rate of fistulization, depending on the technique used [4]. On the contrary, Young obtained excellent results only with the use of a temporoparietal fascial flap [5]. We have not used any type of reconstructive flap, and we have equally obtained a rapid and stable healing of the retroauricular wound.

Finally there is the risk of a iatrogenic (or residual) cholesteatoma. After a SP, it is essential to perform a periodic follow-up, with an annual DWI MRI for at least 5 aa, to exclude the onset of a cholesteatoma. If a cochlear implant has been placed, a CT scan and/or an 1.5 Tesla MRI will be performed.

\section{Conclusions}

$\mathrm{SP}$ is an effective and safe surgical technique to bring the patient to complete healing.

The only defect of an SP may be a slight but significant deterioration of the auditory bone conduction of the $\mathrm{Pt}$, but this problem does not occur when the Pt is already anacusic on that side. SP should be the first choice of treatment in patients with recurrent CSF leakage whenever there is associated unilateral anacusia [6]. This reasoning is fully valid for a Pt with temporal bone fracture and a recurrent meninigitis. In fact otogenic meningitis is a rare but surely life threatening pathology. Every otologist should be able to perform this kind of surgery.

\section{References}

1. Prenzler NK, Gruber L, Lenarz T, Maier H, Schwab B (2018) The Impact of TwoStage Subtotal Petrosectomy and Round Window Vibroplasty on Bone Conduction Thresholds. ORL J Otorhinolaryngol Relat Spec 80: 77-84.

2. Prasad SC, Roustan V, Piras G, Caruso A, Lauda L, et al. (2017) Subtotal petrosectomy: Surgical technique, indications, outcomes, and comprehensive review of literature. Laryngoscope 127: 2833-2842.

3. Casserly P, Friedland PL, Atlas MD (2016) The role of subtotal petrosectomy in cochlear implantation. J Laryngol Otol 130: S35-S40.

4. Lyutenski S, Schwab B, Lenarz T, Salcher R, Majdani O (2016) Impact of the surgical wound closure technique on the revision surgery rate after subtotal petrosectomy. Eur Arch Otorhinolaryngol 273: 3641-3646.

5. Yung M (2016) The Use of Temporoparietal Fascial Flap to Eliminate Wound Breakdown in Subtotal Petrosectomy for Chronic Discharging Ears. Otol Neurotol 37: 248-251.

6. Magliulo G, Iannella G, Ciniglio Appiani M, Re M (2014) Subtotal Petrosectomy and Cerebrospinal Fluid Leakage in Unilateral Anacusis. J Neurol Surg B Skull Base 75 : 391-396. [Crossref]

Copyright: (C2019 Amadei EM. This is an open-access article distributed under the terms of the Creative Commons Attribution License, which permits unrestricted use, distribution, and reproduction in any medium, provided the original author and source are credited. 